\title{
Ultrasound findings of ruptured Peyronie's plaque: Case report and review of the literature
}

\author{
Lucio Dell'Atti ${ }^{1}$, Andrea Benedetto Galosi ${ }^{2}$ \\ ${ }^{1}$ Department of Urology, University Hospital "St.Anna", Ferrara, Italy; \\ 2 Department of Urology, Marche Polytechnic University, Ancona, Italy.
}

\begin{abstract}
Summary We present here a rare case of rupture of tunica albuginea and corpus cavernosum, in the site of a Peyronie's plaque, which happened in a 61year-old man during a vacuum cleaner masturbation.

Ultrasound study showed an irregular hyperechoic defect at the cavernosal rupture site in correspondence of the Peyronie's plaque. The hematoma was evacuated, partially plaque excised, and the tear repaired. Ultrasonography is an ideal technique for evaluating patients with penile trauma and can be routinely used in an emergency. It is a non-invasive method that gives faster results than cavernosography and Magnetic Resonance Imaging.
\end{abstract}

KeY wORDs: Peyronie's disease; Penile trauma; Plaque; Ultrasound; Masturbation.

Submitted 11 December 2016; Accepted 11 January 2017

\section{INTRODUCTION}

We present here a rare case of rupture of tunica albuginea and corpus cavernosum, in the site of a Peyronie's plaque, that happened in a 61-year-old man during a vigorous masturbation. In addition we have reviewed the literature for similar reports and, to our best knowledge, there is the first case reported.

\section{Case report}

A 61-year-old male patient was admitted to our Department of Urology due to the swollen penis with hematoma and penile pain following a sexual act of masturbation with a vacuum cleaner in March 2015.

Approximately 14 months before, due to Peyronie's disease (PD) in the active stage, he had been advised by the urologist to undergo intralesional Verapamil $5 \mathrm{mg}$ one injection/week for a period of 12 weeks and oral medication with vitamin E $400 \mathrm{mg}$ once a day for a period of 18 weeks. His medical history was unblemished, aside from a routine appendectomy when he was 8 year-old and an essential hypertension arose from about 5 years. At the time of our first observation (approximately two hours after the trauma), ultrasound study showed an irregular hyperechoic defect at the cavernosal rupture site in correspondence of a dorsolateral Peyronie's plaque during its stable phase. Ultrasound study of the penis was performed using a General Electric Logiq 7 machine equipped with a high-frequency (7-12.0 MHz) linear transducer. The exact location of the tear has been demonstrated as an interruption of the thin echogenic line of the tunica albuginea and showed an association of hematoma outside dorsal side of the corpora cavernosa (Figure 1). Color-Doppler ultrasound revealed some vascularity at the periphery of the lesion, however the corpus spongiosum was intact, and vascular structures appeared to have a normal flow.

After the informed consent process, the patient was subjected to surgery. The hematoma was evacuated, partially plaque excised, and the tear repaired with prolene 3/0.

In the setting of a dorsal curvature, a short full-thickness vertical incision was made on the ventral shaft tunic opposite the area of maximum curvature, which was then closed transversely to shorten the ventral aspect and correct the curvature. An artificial erection created with intracavernosal injection of normal saline showed an intact repair with no leakages and angulations. The wound was closed with 3/0 vicryl. Duration of hospital stay was 2 days after surgery. After 10 weeks, the patient was able to be sexually active as before. Follow-up with patient occurred every 3 months for 15 months, and did not show any significant angulations of penis.

Figure 1.

B-mode longitudinal ultrasound image shows extra-cavernosal hematoma and the site of ruptured tunica albuginea in correspondence of a dorsolateral Peyronie's plaque.

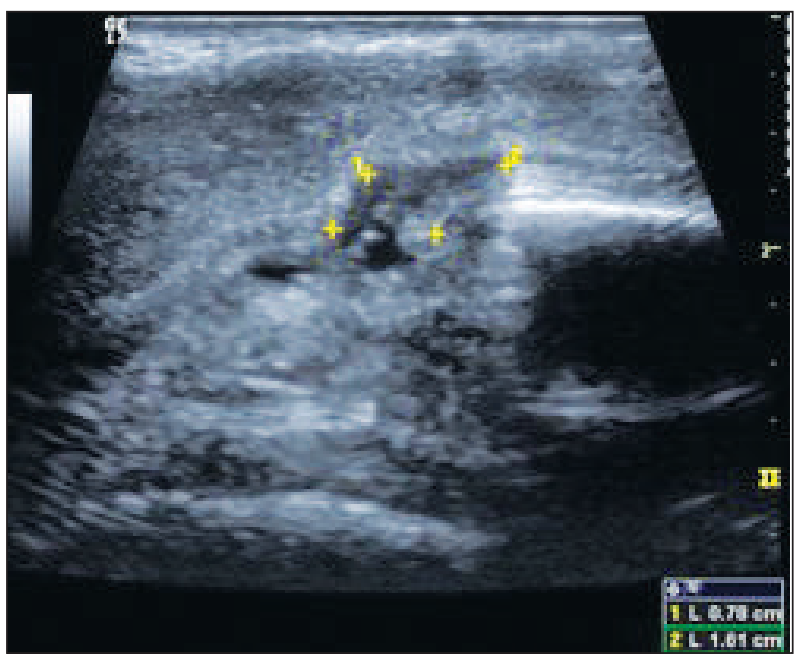




\section{Discussion}

Penile trauma (PT) is a rare urological emergency (1). Usually, it happens in the setting of an erect penis during vigorous sex, masturbation, falls, forceful manipulation, and direct trauma (2). In literature, PT is widely thought to be an important etiology for PD (3-5). PD is defined as a chronic benign fibrotic alteration of the penis of unknown etiology, characterized by the development of plaques or nodules. Occasionally, the patient may be asymptomatic, and the lesion will only be detected by physical examination. In some cases, erectile dysfunction may occur as a result of pain, veno-occlusive dysfunction, or deformity of the penis (4). PD is thought to arise from microvascular trauma during sexual acts leading to inflammation, an aberrant deposition of fibrin, and consequently plaque formation (5). 1980 Hinman proposed that PT could induce mechanical stresses that would lead to plaque formation (6). Devine et al. (7) showed that PD results from acute or repetitive trauma to the penis with microvascular injury. Various factors including genetic predisposition and tissue ischemia have a role in the pathogenesis of the PD (3). Penson et al. (8) observed site specific hemodynamic changes in patients with various degrees of penile trauma and showed that PD may be due to trauma-induced hemodynamic pathology. To our knowledge, however, the association of the PD as a risk factor for occurrence of a PT has never been directly examined let alone proven and there is the first case reported in literature. Probably, in this case study a strong trauma leading to the rupture of the Peyronie's plaque produce a minor resistance in this site due to the accumulation of fibrin and inflammatory cells into the injured area. We hypothesize that an instantaneous and vigorous trauma to the partial erection of penis during a masturbatory act may be cause of the increased mechanical stress associated with the buckling that occurs in patients affected by PD.

Jarow and Lowe (4) demonstrated that the frequency of PT of any kind was significantly greater in both the PD (40\%) and impotence (37\%) patients than in the controls (11\%). However, the reduced incidence of engaging in sexual relations with a partial erection among the PD patients shows that partial impotence is not a predisposing factor for PT (2).

Ultrasonography (US) is an ideal technique for evaluating patients with PT and can be routinely used in an emergency. It permits an evaluation of normal and pathologic anatomic structures (generally smaller than $2 \mathrm{~mm}$ ), and a full evaluation of penile vascularity with sensitivity of color-Doppler to low flow (3). In US, tunica albuginea is easily pictured as a hyperechoic linear line. It can show the integrity of the tunica albuginea, the extent and location of a tunical tear or to quantify the fibrotic involvement caused by the PD. It is very accurate in determining the precise location of the lesions in the penis, as well as the length, width, and thickness of the plaques or to see any hematoma on either side of the tunica (5). Moreover, an ultrasound study is a non-invasive method that gives results faster than cavernosography and Magnetic Resonance Imaging (MRI). However, PD is best viewed in T2-weighted images of MRI (2).

In MRI, Peyronie's plaques are observed as irregularlythickened, low signal intensity areas of tunica albuginea (2). It can be an excellent tissue contrast in evaluation of PT, but it is expensive and not widely available everywhere in emergency. US is operator-dependent and an edematous swelling of the penis within the tear can deteriorate the image contrast, hiding the defect (8).

In our opinion, independently of the presence of a Peyronie's plaque in the rupture site, an immediate surgical treatment is associated with a reduced risk of fibrosis, permanent penile curvature, shorter duration of hospital stay, and decreased return time to normal erectile function.

\section{RefEREnCES}

1. Acikgoz A, Gokce E, Asci R, et al. Relationship between penile fracture and Peyronie's disease: a prospective study. Int J Impot Res. 2011; 23:165-72.

2. Koifman L, Barros R, Júnior RA, et al. Penile fracture: diagnosis, treatment and outcomes of 150 patients. Urology. 2010; 76:1488-92.

3. Joice GA, Burnett AL. Nonsurgical Interventions for Peyronie's Disease: Update as of 2016. World J Mens Health. 2016; 34:65-72.

4. Jarow JP, Lowe FC. Penile trauma: an etiologic factor in Peyronie's disease and erectile dysfunction. J Urol. 1997; 158:1388-90.

5. Levine LA, Burnett AL. Standard operating procedures for Peyronie's disease. J Sex Med. 2013; 10:230-44.

6. Hinman F Jr. Etiologic factors in Peyronie's disease. Urol Int. 1980; 35:407-413.

7. Devine CJ Jr, Somers KD, Jordan SG, et al. Proposal: trauma as the cause of the Peyronie's lesion. J Urol 1997; 157:285-90.

8. Penson DF, Seftel AD, Krane RJ, et al. (1992) The hemodynamic pathophysiology of impotence following blunt trauma to the erect penis. J Urol. 1992; 148:1171-80.

\section{Correspondence}

Lucio Dell'Atti, MD, PhD (Corresponding Author)

dellatti@hotmail.com

Department of Urology, University Hospital "St.Anna"

8 A. Moro Street - 44124 Cona, Ferrara, Italy

Andrea Benedetto Galosi, MD

Department of Urology, Marche Polytechnic University

Ancona, Italy 